\title{
Análise crítica dos métodos utilizados para determinar o surto de crescimento puberal pelos especialistas em ortodontia
}

\author{
Eduardo José Carvalho de Souza-Junior ${ }^{1}$ \\ Regina Cerqueira Wanderley-Cruz ${ }^{2}$
}

\begin{abstract}
Resumo
Avaliar o surto de crescimento puberal (SCP) é importante na prática clínica ortodôntica, visto que o crescimento corporal que acontece nessa fase pode atuar como um excelente coadjuvante no tratamento. Esse estudo se propôs a analisar quais e como os métodos são utilizados pelos especialistas em ortodontia, da cidade de Salvador (Bahia), para avaliar o SCP. Foram enviados questionários com perguntas fechadas e abertas a todos os dentistas registrados no CROBA como especialistas em ortodontia. $\mathrm{O}$ universo foi composto de 90 ortodontistas e, desses, 67 responderam o questionário, constituindo uma amostra em que $64,1 \%$ dos informantes pertenciam ao sexo feminino. Os resultados mostram que todos os entrevistados concordaram ser necessário verificar o SCP e, desses, $94 \%$ indicaram seu uso para casos específicos como: pacientes em crescimento, distúrbios de crescimento ósseo, tratamento ortocirúrgico e (ou) ortopédicos, casos limítrofes, dúvidas sobre o estágio de desenvolvimento. Quanto ao método utilizado, houve uma predominância da análise carpal, seguida da análise das vértebras cervicais, dental e caracteres sexuais secundários. Nenhum dos entrevistados vinculou o SCP apenas à idade cronológica. Entretanto, quando solicitados a listar nomes dos métodos preferidos e dificuldades encontradas, foi grande o número de entrevistados que deixou de responder as perguntas. Tal fato faz concluir ser necessária uma maior divulgação dos métodos de análise do SCP, principalmente da análise das vértebras cervicais, que é o mais recente.
\end{abstract}

Palavras-chave: Idade dentária - Métodos de avaliação - Análise radiográfica - Idade óssea - Métodos de avaliação Análise radiográfica - Surto de crescimento puberal - Métodos de análise.

\section{INTRODUÇÃO}

A determinação do estágio de crescimento e de desenvolvimento do paciente é essencial para direcionar o tratamento clínico pelo cirurgião-dentista, em especial o ortodontista. $\mathrm{Na}$ adolescência, ocorrem alterações morfofuncionais significativas em relação ao crescimento do corpo humano, e o período de maior aceleração no desenvolvimento corpóreo chama-se surto de crescimento puberal ${ }^{1,2}$. Esse surto oferece vantagens para certos tipos de tratamento e procedimentos odontológicos, pois envolve todas as dimensões musculares e ósseas, tornando o tratamento ortodôntico e ortopédico mais biológico que mecânico ${ }^{3,4}$. Para Martins e

\footnotetext{
${ }^{1}$ Mestrando em Clínica Odontológica (Dentística)- UNICAMP.

${ }^{2}$ Professora de Radiologia Clínica - Faculdade de Odontologia - UFBA; Doutora em Estomatologia, UFBA/UFPB.

Correspondência para / Correspondence to:

Eduardo José Carvalho de Souza-Junior

Rua Prof. Cassilandro Barbuda, 487, apt. 201 - Costa Azul.

41760-110 Salvador - Bahia - Brazil.

Tel: (71) 8203-5085.

E-mail: edujcsj@gmail.com
} 
Sakima ${ }^{5}$, trata-se de uma curva de crescimento que relaciona estágios de maturação óssea com a idade cronológica, altura corporal, caracteres sexuais secundários, em que, no primeiro momento, ocorre um incremento até se chegar ao ápice, o chamado pico do surto de crescimento e, depois, há um decréscimo até parar. Fatores genéticos e ambientais diversificam a cronologia dos eventos de crescimento individual ${ }^{6}$, fazendo-se necessária uma determinação competente desse surto de crescimento puberal (SCP) e da maturação do individuo, o que é importante para o êxito de tratamentos que contam com o crescimento do ser humano como auxiliar. A idade cronológica, o desenvolvimento dentário e esquelético, a altura, o peso e a manifestação dos caracteres sexuais secundários constituem alguns parâmetros que são utilizados para a mensuração do grau de crescimento de um indivíduo. ${ }^{7,8}$

A idade esquelética é o indicador mais utilizado para a determinação do crescimento de um indivíduo, por ser mais fidedigna no esclarecimento dos estágios de desenvolvimento físico do ser humano. Entretanto, outros parâmetros também são aplicados, muitas vezes em conjunto. Vários estudos têm revelado métodos considerados eficazes, com validez comprovada, para a avaliação do surto de crescimento puberal, sendo os mais difundidos a análise carpal 2,3,4,5,6,9,10, os estágios de desenvolvimento dos dentes ${ }^{11,12}$ e a comparação entre os dois métodos ${ }^{1,13,14,15,16,17}$. O método mais amplamente utilizado é a radiografia da mão e punho, 2,18,19,20,21,22,23, por concentrar uma variedade de centros de ossificação na mesma tomada radiográfica e ser de fácil execução, o que pode ser feito comparando-se a radiografia carpal do paciente com o Atlas proposto por Greulich e Pyle ${ }^{24}$. Alguns estudos sugerem um método mais simplificado para avaliar a maturação esquelética, mediante o uso de películas radiográficas periapicais, para a verificação da presença do osso sesamóide da articulação metacarpo falangeana do dedo polegar. ${ }^{25,26}$

Trabalho clássico sobre os estágios de desenvolvimento dos dentes, foi desenvolvido por Nolla ${ }^{11}$, que propôs uma classificação com 10 estágios que podem ser subdivididos, facilitando o cálculo da idade dental. Também Demirjian, Goldstein e Tanner ${ }^{12}$, elaboraram um estudo no qual as fases desenvolvimento dos dentes foram divididas em 8 e permitiram o cálculo da idade dental.

Recentemente, a análise da forma das vértebras cervicais C2 (processo odontóide), C3 e C4 em telerradiografias em norma lateral tem sido usada para determinar a expectativa de crescimento e desenvolvimento do indivíduo $8,27,28,29,30,31,32,33$ e também para determinar anomalias de desenvolvimento ósseo dessas estruturas ${ }^{28,29}$. Esse método tem como principal vantagem o fato de poder ser executado a partir de telerradiografias em norma lateral, as quais já são utilizadas para o diagnóstico das maloclusões na clínica ortodôntica, evitando-se, assim, uma exposição desnecessária do paciente aos raios $\mathrm{X}^{7,31,34,35}$. O método da análise da forma das vértebras cervicais em telerradiografias é maior para o de Lamparski, de 1972, modificado por Hassel e Farman ${ }^{27}$, em 1995, que é também o mais divulgado.

Nesse panorama tão diverso, em que o cirurgião-dentista pode escolher o método que considerar mais conveniente, conhecer a opinião dos ortodontistas de Salvador (BA) sobre esses métodos estabelecerá um quadro do conhecimento dos especialistas, das dificuldades encontradas pelos profissionais e, além disso, das soluçōes que podem ser sugeridas para resolver esses problemas.

Para tanto, este estudo se propôs a analisar os métodos que são utilizados pelos ortodontistas especialistas, da cidade de Salvador ( Bahia), em 2005, para determinar o surto de crescimento puberal.

\section{MATERIAIS E MÉTODOS}

Foi realizado um estudo transversal, com amostra de conveniência, onde foram enviados questionários, com perguntas de múltipla escolha e abertas, a todos os cirurgióes-dentistas registrados no Conselho Regional de Odontologia da Bahia, até o dia 5 de agosto de 2004, como especialistas em ortodontia, da cidade de Salvador, num total de 90. Os 
questionários foram enviados pelo correio ou entregues diretamente ao especialista, marcandose o retorno para receber o questionário respondido. Em anexo ao questionário, havia um termo de consentimento esclarecido, informando a finalidade da pesquisa, que garantia o anonimato e o direito de abandonar a pesquisa a qualquer momento. $\mathrm{O}$ período de aplicação do questionário foi de outubro de 2004 a maio de 2005.

O projeto da pesquisa foi aprovado pela Comissão de Ética da Faculdade de Odontologia da Universidade Federal da Bahia. Recebidos os questionários, os resultados foram tabulados e foi realizada uma análise descritiva em software Excel 2000 for Windows Xp.

\section{RESULTADOS E DISCUSSÃO}

A busca por planejamento de tratamentos mais adequados em Ortodontia perpassa pela necessidade de conhecer a idade biológica do paciente, pois o fator crescimento pode tanto implicar um tratamento adequado, como também, o modo como esse conhecimento é utilizado a favor de um melhor resultado clínico. A análise da idade esqueletal é considerada o fator preponderante para a determinação do crescimento e do desenvolvimento, sendo a análise da radiografia dos ossos da mão e do punho, a chamada análise carpal, a mais utilizada, existindo diversos métodos para realizar essa análise, cada um com características próprias ${ }^{2,3,4,5,6,9,10}$. Além da análise carpal, a análise das vértebras cervicais começa a ganhar espaço na prática clínica odontológica, pela praticidade, pelo fato de utilizar uma radiografia que já é realizada para o planejamento e consequentemente menor exposição de raios $\mathrm{X}$ e diminuição do custo para o paciente. ${ }^{8,27,28,29,30,31,32,33}$

Outros métodos para análise de desenvolvimento, como a idade dental e os caracteres sexuais secundários, também são frequentemente usados associados ou não à idade óssea. ${ }^{1,11,12,15,17}$

Nosso estudo teve como universo 90 especialistas em ortodontia da cidade de Salvador, que constituíram uma amostra de conveniência, e o percentual de retorno dos questionários foi de 74,4\%, correspondendo a 67 entrevistados. A amostra foi constituída de $64,2 \%$ do sexo feminino, e $35,8 \%$ do sexo masculino.

Quando questionados sobre a necessidade do uso de um método para analisar o surto de crescimento puberal, todos os entrevistados (100\%) responderam afirmativamente. Desses, apenas 3\% fazem uso de método de análise do SCP em todos os pacientes; os outros $94 \%$ indicam a análise do SCP apenas em pacientes em fase de crescimento com ou sem distúrbio, nos casos em que a avaliação do crescimento interferirá no planejamento do tratamento e no diagnóstico para tratamento ortocirúrgico e (ou) ortopédicos. Tais respostas mostram o critério dos entrevistados na indicação da análise de crescimento. Importante observar uma contradição, pois, apesar de terem respondido considerar importante avaliar o SCP, 3\% dos entrevistados não fazem uso de qualquer análise para avaliar o SCP, de forma que as demais perguntas ficaram sem resposta, diminuindo o número da amostra para 65 especialistas.

Em relação aos métodos mais utilizados para análise do SCP, foi possível selecionar mais de uma resposta, uma vez que muitas pesquisas mostram a associação de métodos para se chegar ao diagnóstico ${ }^{8,27,30,31,32}$. Dessa forma, foram encontrados os seguintes percentuais: carpal (97\%), caracteres sexuais secundários (63\%), análise dos dentes (46\%), vértebras cervicais (34\%) e idade cronológica (31\%) (Gráfico 1). A maior utilização do método carpal provavelmente se deve ao fato de ser um método de análise muito antigo ${ }^{24}$, além de ser amplamente divulgado na literatura, com diversos trabalhos. $2,3,4,5,6,9,10$

No item seguinte, questionava-se como a análise carpal é feita, uma vez que hoje é grande o número de clínicas de Radiologia que oferecem esse serviço, como também existem profissionais que preferem fazer sua própria análise. Nesse item, observou-se uma predominância por clínicas especializadas, com $73 \%$, seguida por $18 \%$ de profissionais que preferem fazer sua própria análise, enquanto que $9 \%$ não têm preferência (Gráfico 2). Esse resultado indica a 
Gráfico 1. Distribuição dos indivíduos em relação aos métodos utilizados para avaliar o surto de crescimento puberal (SCP).

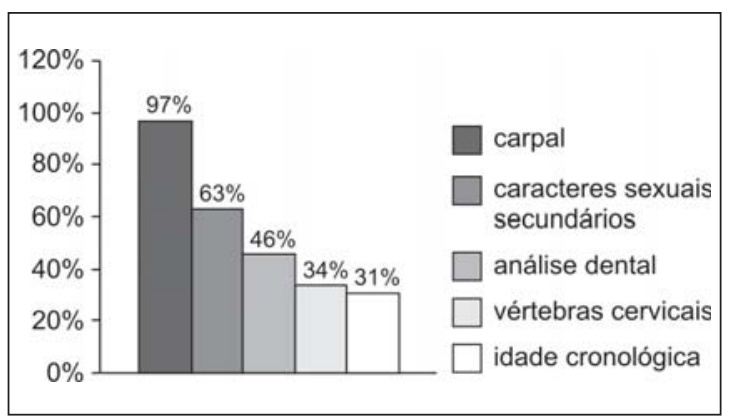

Gráfico 2. Distribuição do modo de realização do método carpal.

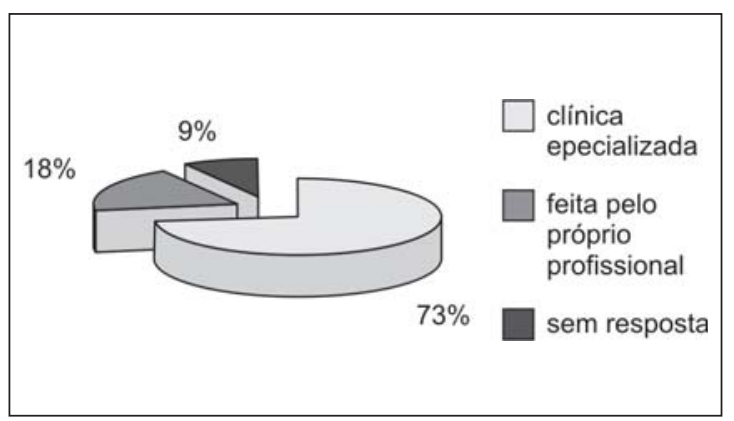

Gráfico 3. Distribuição quanto às dificuldades do método carpal.

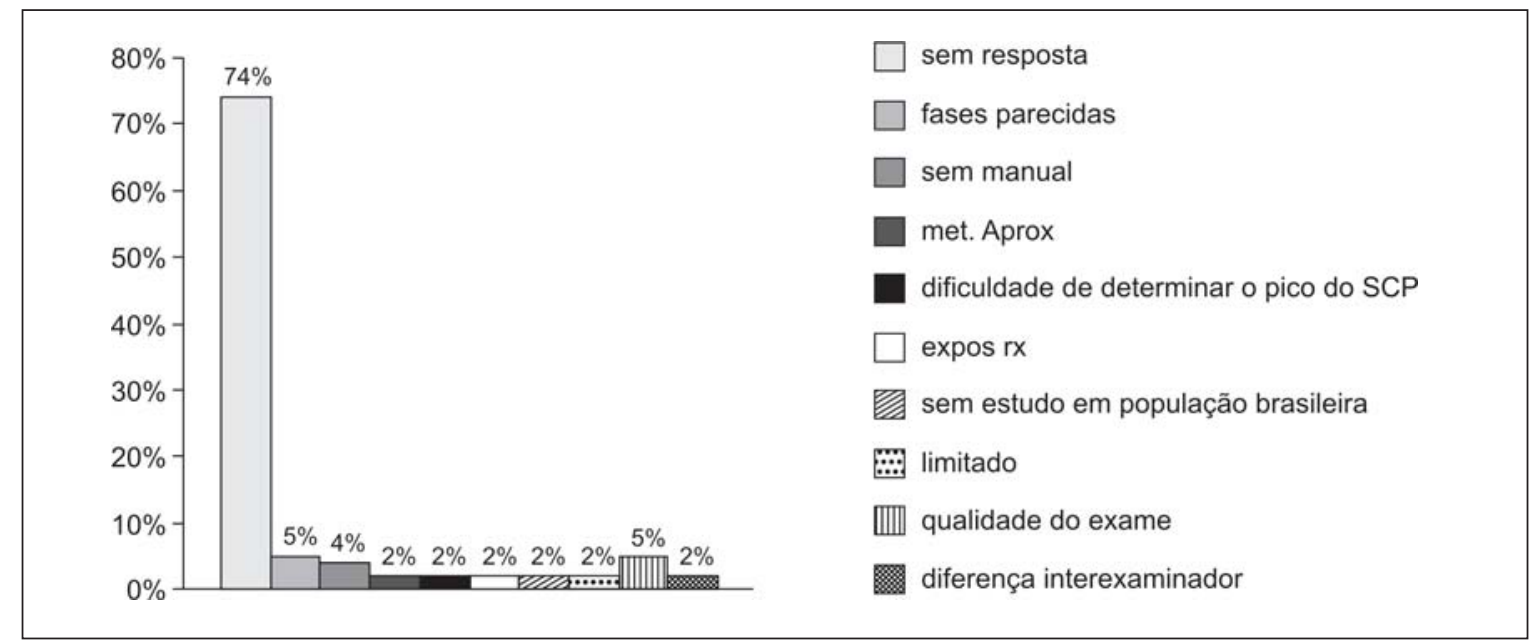

Gráfico 4. Distribuição quanto aos métodos carpais utilizados.

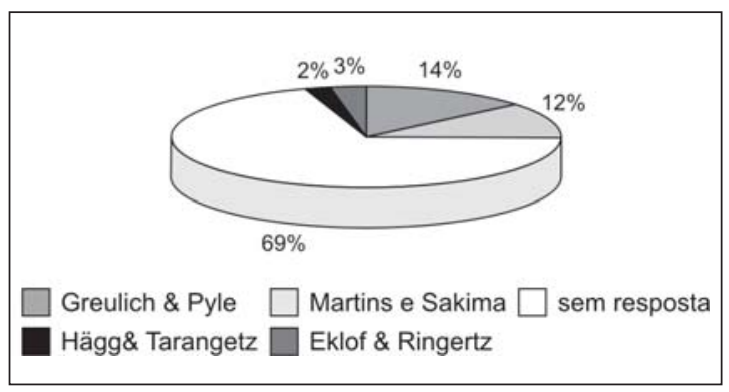

tendência atual de terceirizar serviços de forma a otimizar o tempo no consultório. Essa tendência é reforçada na questão seguinte, pois, ainda em relação à forma como é feita a análise, foi perguntado se ela deveria ser manual ou pelo
Gráfico 5. Distribuição quanto às dificuldades do método das vértebras cervicais.

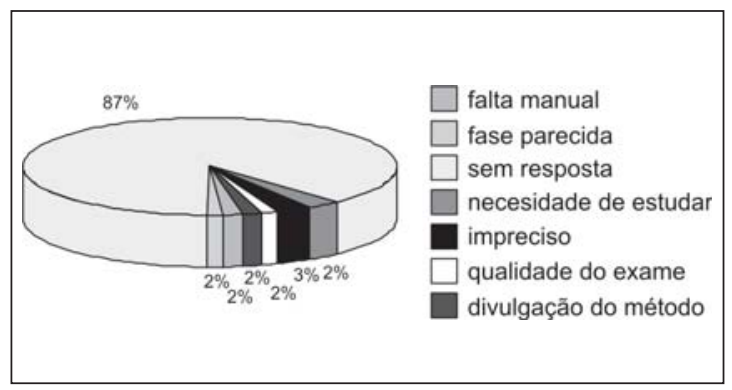

computador, e $68 \%$ consideraram que a análise deve ser computadorizada.

Ao se verificar a compreensão do método carpal, $68 \%$ dos entrevistados relataram que a análise carpal é de fácil compreensão. 
Entretanto, quando questionados a listar as dificuldades encontradas, o índice de questionários sem resposta foi de $74 \%$. Tal percentual de abstenção pode estar associado a duas hipóteses: pelo fato de o método ser altamente divulgado e utilizado há bastante tempo, pode-se ter um grau maior de conhecimento do tema, o que faz com que os entrevistados não encontrem dificuldades; ou ainda, uma segunda hipótese: o fato de se fazer uso em maior índice das análises computadorizadas, que já vem com provável diagnóstico, facilitando a compreensão do método. Porém essas hipóteses não foram testadas. Entre os que responderam essa questão, foram listadas as seguintes dificuldades com os respectivos percentuais: fases muito parecidas $(5 \%)$, qualidade da radiografia $(5 \%)$, necessidade da existência de um manual para orientação diagnóstica $(4 \%)$, diferença de interpretação interexaminadores $(2 \%)$, método aproximado (2\%), método limitado (2\%), dificuldade na determinação exato do SPC (2\%), exposição adicional aos raios $X(2 \%)$, não possuir estudo para a população brasileira (2\%) (Gráfico 3).

Nesse caso, cabem algumas indagaçōes, que podem ser alvo de novos estudos: será que todos os métodos têm algumas fases muito parecidas? A causa citada ocorreu devido à diferença interexaminadores? Se a maioria prefere análises computadorizadas, será que essa diferença tem a ver com o programa utilizado? Quanto interfere no planejamento, realiza-se a determinação exata do pico de crescimento puberal? Será que essa dificuldade listada se deve ao fato de ser necessária maior divulgação?

Não é verdadeiro que não existam trabalhos feitos na população brasileira com o método carpal. 2,5,6,10

Um dado importante encontrado é o fato de $55 \%$ dos entrevistados questionarem os resultados da análise carpal; porém, nas respostas anteriores, apenas 26\% dos entrevistados listaram dificuldades, sugerindo inclusive o uso de um manual diagnóstico (5\%), e o método é altamente utilizado pelos especialistas (97\%). Quando questionados sobre que método de análise carpal é o mais empregado por eles: 14\% citaram Greulich e Pyle, 12\% Martins e Sakima,
3\% Eklof e Ringertz, 2\% Hägg e Tarangetz. Os outros 69\% não responderam (Gráfico 4).

Sobre a análise das vértebras cervicais para diagnóstico do surto de crescimento puberal, observou-se que 63\% dos especialistas afirmaram conhecer o método das vértebras cervicais, e apenas 23\% dos entrevistados afirmaram já ter aplicado esse método. Trata-se de um método cuja divulgação é mais recente, com um aumento de estudos a partir de 1995, pois, no Brasil, os trabalhos têm inicio a partir de 2001, o que se mostra fidedigno pela literatura $8,27,29,30,31,32,33$. Tais fatos explicam os resultados acima.

Dentre os entrevistados, $72 \%$ acreditam que o método das vértebras cervicais é de fácil compreensão, com uma discrepância em relação à questão anterior, na qual $63 \%$ dizem conhecer o método. Ao serem convidados a listar as dificuldades desse método, a grande maioria dos entrevistados não respondeu (87\%), apesar de $23 \%$ dos especialistas já terem aplicado o método. As dificuldades listadas foram as seguintes: fases muito parecidas (2\%), necessidade de um protocolo em anexo para ajudar no diagnóstico (2\%), falta de estudo para a interpretação do método (2\%), imprecisão $(3 \%)$, qualidade dos exames $(2 \%)$ e pouca divulgação do método (2\%) (Gráfico 5). Reafirma-se, aqui, o fato de ser uma análise aplicada recentemente, havendo necessidade de maior divulgação do método com a elaboração de protocolos que auxiliem os especialistas no diagnóstico.

Considerada como uma forma de análise do SCP, a análise dos dentes foi avaliada através de questões como forma de aplicação, e as respostas foram: complementar aos dados clínicos $(7 \%)$, pela avaliação dos estágios de formação dental na radiografia $(7 \%)$, pelo grau de erupção dental na radiografia $(2 \%)$, a associação de duas respostas (17\%) e associação de todas as alternativas (67\%). A análise dos dentes para determinação da idade tem como marco a pesquisa de Nolla ${ }^{11}$, permitindo a validação desse e de outros estudos com esse tema ao longo dos anos. Trata-se, portanto, de um conhecimento adquirido profundo que permite aos entrevistados responder com 
segurança sobre a forma de utilização, pois a somatória das formas de aplicação é a mais frequente.

As maiores dificuldades encontradas pelos profissionais para a realização do método de análise dental foram: definir o que é coroa completa de $1 / 3$ de raiz formada (2\%), radiografias que não têm boa visualização das raízes com rizogênese incompleta $(2 \%)$, além da dificuldade de determinar o estágio quando o indivíduo tem desenvolvimento muito precoce (2\%). Os outros $96 \%$ dos entrevistados não responderam.

Em relação ao diagnóstico muitas vezes confuso de coroa completa ou de $1 / 3$ de raiz formada (estágio aplicado somente na análise de Nolla) e, além disso, a dificuldade de determinar o estágio quando o individuo tem um desenvolvimento precoce fazem com que haja necessidade de aprofundamento da análise feita, com uma nova calibração do examinador. Quanto à qualidade das radiografias, é preciso avaliar se a dificuldade está associada ao paciente ou não, e buscar formas para suprir esse empecilho com alternativas específicas para cada caso.

O método mais utilizado para avaliar a idade dental foi o de Nolla (84\%), observandose ainda o método de Demirjian $(2 \%)$ e o de Nicodemo-Moraes-Médici Filho (2\%). Houve $12 \%$ sem resposta a esse questionamento (Gráfico 6).

Outro método citado como parâmetro para avaliação do SCP, os caracteres sexuais

Gráfico 6. Distribuição em relação aos tipos de análise da idade dental.

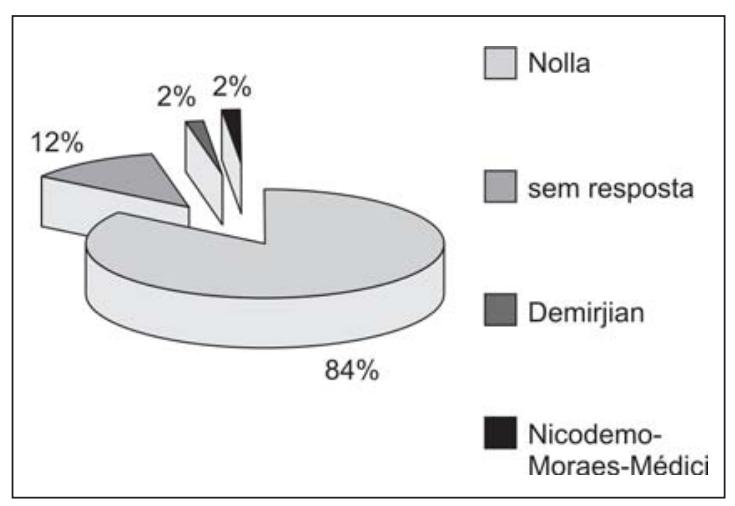

secundários, ele é considerado por $85 \%$ dos entrevistados como uma análise válida somente quando associada a outros métodos, enquanto $6 \%$ afirmam ser um parâmetro falho e $9 \%$ consideram uma boa análise (Gráfico 7). Tratase de método amplamente divulgado, de conhecimento geral e que não gera muitas dúvidas, sendo sempre empregado como método auxiliar.

Gráfico 7. Distribuição em relação à opinião quanto aos caracteres sexuais secundários.

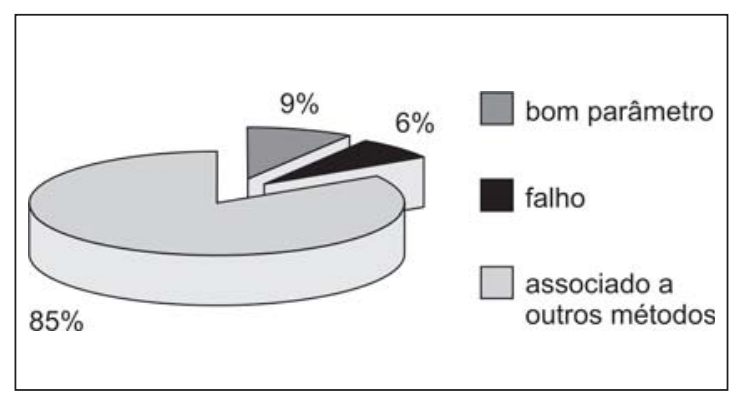

Esta pesquisa teve um alto índice de retorno dos questionários, refletindo o pensamento da maioria dos especialistas em ortodontia da cidade de Salvador, registrados no CROBA. Percebe-se, graças a ela, que existe um conhecimento maior sobre os métodos de análise do surto de crescimento puberal em relação à análise carpal, dos dentes e dos caracteres sexuais secundários, com análise crítica sobre as dificuldades encontradas em cada método. Em relação à análise das vértebras cervicais, esse conhecimento ainda é pequeno, embora já exista um juízo de valor sobre o assunto.

\section{CONCLUSÕES}

Considerando os métodos de avaliação do surto de crescimento puberal, a aplicação e o conhecimento do tema, concluiu-se que:

- a análise carpal é a mais utilizada, existindo grande conhecimento sobre o tema e uma análise crítica por parte dos profissionais; 
- as análises dos caracteres sexuais secundários e dos dentes são aplicadas como coadjuvantes de outros métodos, com conhecimento e sem muitas dúvidas sobre ao assunto;
- em relação à análise das vértebras cervicais, é necessária maior divulgação do método, de forma a permitir uma aplicação com segurança pelos ortodontistas.

\title{
Critical analysis of the methods used to evaluate pubertal growth spurt by the specialists in orthodontics
}

\begin{abstract}
Evaluation of pubertal growth spurt (PGS) is important in orthodontic dental practice, because body development that happens at this time of life, can act as an excellent collaborator in dental treatment. The aim of this study is to analyse the methods and how they are used by the specialists in orthodontics, in Salvador - Bahia, to evaluate PGS. Questionnaires were sent with objective and subjective questions to all specialist in orthodontics registered in CROBA. From all 90 orthodontists, only 67 answered, being the convenience sample in which $64,1 \%$ were female. The results showed that all orthodontists agree to be necessary to verify the PGS and, $94 \%$ had indicated its use for specific cases like: patients in growth, disturbances of osseous growth, orthodontic-surgery treatment andlor ortophaedic, bordering cases, doubts on the period of development. About the used method, it had a predominance of hand and wrist analysis, followed by the cervical vertebrae analysis, dental method and secondary sexual characters. None of the specialists related PGS only to the chronological age. However, when requested names of the preferred methods and difficulties found, there was a large number of specialists who did not answer the questions. Such fact makes to conclude that it is necessary greater spreading of the methods to analyse the PGS, specially the analysis of the cervical vertebrae method, which is most recent.
\end{abstract}

Keywords: Dental age - Evaluation methods - Radiographic analysis; Skeletal maturation - Evaluation methods - Radiographic analysis; Pubertal growth spurt - Evaluation methods.

\section{REFERÊNCIAS}

1 MORAES, M.E.L; MÉDICI FILHO, E.; MORAES, L.C. Surto de crescimento puberal: relação entre mineralização dentária, idade cronológica, idade dentária e idade óssea: método radiográfico. R. Odontol. UNESP, Marília, v.27, n.1, p.111-129, 1998.

2 CHAVES, A.P.; FERREIRA, R.I.; ARAÚJO, T.M. Maturação esquelética nas raças branca e negra. Ortodon. Gauch., Porto Alegre, v.3, n.1, p.45-52, 1999.

3 GRAVE, K.C.; BROWN, T. Skeletal ossification and the adolescent growth spurt. Am. J. Orthod., St.Louis, v.69, n.6, p.611-619, 1976.
4 FISHMAN, L.S. Radiografic evaluation of skeletal maturation: a clinically oriented method based on hand-wrist films. Angle Orthod., Appleton, v.52, n.2, p.88-112, 1982.

5 MARTINS, J.C.R.; SAKIMA, T. Considerações sobre a previsão do surto de crescimento puberal. Ortodontia, São Paulo, v.10, n.3, p.164-170, 1977.

6 TIBÉRIO, S.; VIGORITO, J.W. O estudo da maturação esquelética de crianças brasileiras leucodermas, de 8 a 15 anos, em referência à ossificação dos ossos pisiforme, ganchoso, falanges média e proximal dos dedos 2 e 3 . Ortodontia, São Paulo, v.22, n.2, p.4$19,1989$. 
7 SANTOS, S.C.B.N. et al.. Avaliação de um método de determinação do estágio de maturação esquelética utilizando as vértebras cervicais presentes nas telerradiografias em norma lateral. R. Dent. Press Ortodon. Ortop Facial, Maringá, v.3, n.3, p.67-79, 1998.

8 ARMOND, M.C.; CASTILHO, J.C.M.; MORAES, L.C. Estimativa do surto de crescimento puberal pela avaliação das vértebras cervicais em radiografiascefalométricas laterais. Ortodontia, São Paulo, v.34, n.1, p.51-60, 2001.

9 BJORK, A.K.; HELM, S. Prediction of the age of maximum puberal growth in body height. Angle Orthod., Appleton, v.37, n.2, p.134-143, 1967.

10 IGUMA, K.E.; TAVANO, O.; CARVALHO, I.M.M. Comparative analysis of pubertal growth spurt predictors: Martins and Sakima method and Grave and Brown method. J. Appl. Oral Sci., Bauru, v.13, n.1, p.58-61, 2005.

11 NOLLA, C.M. The development of the permanent teeth. J. Dent. Child., Fulton, v.2, p.254-266, 1960.

12 DEMIRJIAN, A.; GOLDSTEIN, H.; TANNER, J.M. A new system of dental age assessment. Hum. Biol., Detroit, v.45, n.2, p.211-227, 1973.

13 SO, L.L.Y. Skeletal maturation of the hand and wrist and its correlation with the dental development. Aust. Orthod. J., Brisbane, v.15, n.1, p.1-9, 1997.

14 UYSAL, T. et al. Relationships between dental and skeletal maturity in Turkish subjects. Angle Orthod., Appleton, v.75, n.4, p.657664, 2004.

15 ETO, L.F.; MAZZIEIRO, E.T. Avaliação da correlação entre os estágios de mineralização dos dentes inferiores e a idade esquelética observados sob o gráfico de crescimento puberal. R. Dent. Press Ortodon. Ortop Facial, Maringá, v.10, n.2, p.75-86, 2005.

16 FLORES-MIR, C. et al. Association between growth stunting with dental development and skeletal maturation stage. Angle Orthod., Appleton, v.75, n.6, p.935-940, 2005.
17- FLORES-MIR, C. et al. Correlation of skeletal maturation stages determined by cervical vertebrae and hand and wrist evaluations. Angle Orthod., Appleton, v.76, n.1, p.1-5, 2006.

18 MAPPES, M.S.; HARRIS, E.F.; BEHRENTS, R.G. An example of regional variation in the tempos of tooth mineralization and hand-wrist ossification. Am. J. Orthod. Dentofac. Orthop., St. Louis, v.101, n.2, p.145-151, 1992.

19 FRANCO, A.A. et al. Determinação radiográfica da maturidade esquelética e sua importância no diagnóstico e tratamento ortodôntico. Ortodontia, São Paulo, v.29, n.1, p.53-59, 1996.

20 DIAS, P.P.F.; GUSMÃO, J.M.R.; ARAÚJO, T.M. Avaliação da idade esquelética. J. CEO, Salvador, v.2, n.4, p.3, 1998.

21 CORDEIRO, R.C.L. et al. Determinação da idade óssea, estatura e peso de crianças da zona rural da cidade de Araraquara. J. Bras. Ortodon. Ortop. Facial, Curitiba, v.4, n.24, p.469-475, 1999.

22 GUZZI, B.S.S.; CARVALHO, L.S. Estudo da maturação óssea em pacientes jovens de ambos os sexos através de radiografias de mão e punho. Ortodontia, São Paulo, v.33, n.3, p.49-58, 2000.

23 MORA, S. et al. Skeletal age determinations in children of European and African descent: applicability of the Greulich and Pyle standards. Pediatr. Res., Hagerstown, n.50, n.5, p.624628, 2001.

24 GREULICH, W.W.; PYLE, S.I. Radiographic atlas of skeletal development of the hand and wrist. $2^{\text {nd }}$.ed. Palo Alto: Stanford University Press, 1959.

25 SILVA FILHO, O.G.; SAMPAIO, L.L.; FREITAS, J.A.S. Avaliação de um método simplificado para estimar a maturação esquelética. Ortodontia, São Paulo, v.25, n.1, p.21-36, 1992.

26 MORI, G.G. et al. Avaliação da eficácia do método simplificado a partir de comparação com o método de Fishman, para a determinação da idade esquelética.R. Paul. Odontol., São Paulo, v.23, n.4, p.29-31, 2001. 
27 HASSEL, B.; FARMAN, A.G. Skeletal maturation evaluation using cervical vertebrae. Am. J. Orthod. Dentofac. Orthop., St. Louis, v.107, n.1, p.58-66, 1995.

28 O'REILlY, M.T.; YANNIELLO, G.J. Mandibular growth changes and maturation of cervical vertebrae: a longitudinal cephalometric study. Angle Orthod., Appleton, v.58, n.2, p.179-184, 1988.

29 VASTARDIS, H.; EVANS, C.A. Evaluation of cervical spine abnormalities on cephalometric radiographs. Am. J. Orthod. Dentofac. Orthop., St. Louis, v.109, n.6, p.581-588, 1996.

30 WANDERLEY-CRUZ, R.C. Maturação das vértebras cervicais e desenvolvimento dentário em indivíduo de oito a quinze anos de idade. 2002. Tese (Doutorado) - Faculdade de Odontologia, Universidade Federal da Bahia, Salvador, 2002.

31 CANALI, L.; BRÜCKER, M.R.; LIMA, E.M.S. Avaliação da maturação esquelética das vértebras cervicais através de telerradiografias de perfil. R. Odonto Ciênc., Porto Alegre, v.18, n.40, p.127-137, 2003.
32 GENEROSO, R. et al. Estudo da correlação entre a idade cronológica e a maturação das vértebras cervicais em pacientes em fase de crescimento puberal. R. Dent. Press Ortodon. Ortop Facial, Maringá, v.8, n.4, p.19-36, 2003.

33 SANTOS, E.C.A. et al. Avaliação da reprodutibilidade do método de determinação da maturação esquelética por meio das vértebras cervicais. R. Dent. Press Ortodon. Ortop Facial, Maringá, v.10, n.2, p.62-68, 2005.

34 BACCETTI, T.; FRANCHI, L.; McNAMARA JUNIOR, J.A. An improved version of the cervical vertebrae maturation (CMV) method for the assessment of mandibular growth. Angle Orthod., Appleton, v.72, n.4, p.316-323, 2002 .

35 CHEN, F; TERADA, K.; HANADA, K. A new method of predicting mandibular length increment on the basis of cervical vertebrae. Angle Orthod., Appleton, v.74, n.5, p.630634, 2004. 